\title{
In-situ antenna diagnostics and characterization system based on RFID and remotely piloted aircrafts
}

\author{
Yuri Álvarez López ${ }^{\mathrm{a}, *}$, María García Fernández ${ }^{\mathrm{a}}$, Guillermo Álvarez Narciandia, Ana Arboleya Arboleya \\ «a,b, Fernando Las-Heras Andrés ${ }^{a}$, Silverio García Cortés ${ }^{c}$, Manés Fernández Cabanas ${ }^{\mathrm{d}}$ \\ ${ }^{a}$ Área de Teoría de la Señal y Comunicaciones, Departamento de Ingeniería Eléctrica, Universidad de Oviedo, Spain. \\ ${ }^{b}$ Polytech' Lab, University Nice Côte d'Azur, Campus Sophia-Tech, France. \\ ${ }^{c}$ Departamento de Explotación y Prospección de Minas, Escuela politécnica de Mieres, Universidad de Oviedo, Spain. \\ ${ }^{d}$ Área de Ingeniería Eléctrica, Departamento de Ingeniería Eléctrica, Universidad de Oviedo, Spain.
}

\begin{abstract}
A cost-effective solution for in-situ antenna characterization and diagnostics based on Radio Frequency IDentification technology and Remotely Piloted Aircrafts is presented. The cost-effectiveness of the proposed solution is achieved by replacing the radio frequency equipment on-board the small aircrafts with Radio Frequency IDentification tags, while the Antenna Under Test is connected to a Radio Frequency IDentification reader, thus reducing the weight and complexity of the payload of the aircrafts dedicated to antenna measurement task. Received Signal Strength measurements are geo-referred with centimeter-level accuracy thanks to a Real Time Kinematic system. An iterative phase retrieval technique based on the Sources Reconstruction Method is used to recover an equivalent magnetic currents distribution on the Antenna Under Test aperture plane. The reconstructed currents distribution provides antenna diagnostics information and enables the calculation of the antenna radiation pattern. The presented method has been validated by means of measurements in the UHF band for two different antenna arrays with excellent results.
\end{abstract}

Keywords: Antenna diagnostics, Received Signal Strength (RSS), Radio Frequency Identification (RFID), Antenna radiation pattern, Remotely Piloted Aircraft (RPA), Real Time Kinematic (RTK).

\section{Introduction}

The great cost reduction of the fabrication technology of small Remotely Piloted Aircrafts (RPAs) has motivated the development of new types of applications in fields such as civil engineering [1, 2, agriculture 3. 4], mining [5], etc.

Although less accurate than anechoic-chamber measurements, in-situ antenna characterization can provide useful information about antenna performance in its final environment while being a less expensive

\footnotetext{
Ana Arboleya was with Universidad de Oviedo when the measurement campaign was done but she is currently part of the Polytech' Lab in University of Nice Côte d'Azur.

${ }^{*}$ Corresponding author

Email address: yalopez@tsc.uniovi.es (Yuri Álvarez López )
} 
technique. Thanks to the advances in RPA instrumentation and the availability of Real-Time Kinematic (RTK) hardware capable of providing centimeter-level positioning accuracy, the use of RPAs for in-situ antenna characterization has proven to be more accurate than traditional land-based solutions or manned aircraft based surveys for in-situ antenna characterization [6].

There are several RPA-based systems available in the literature that have been successfully validated for antenna characterization. In [6] an antenna and a receiver (RX) are installed on-board an RPA for the direct far-field (FF) characterization of high-powered broadcast antenna systems by means of amplitudeonly measurements. The weight of the complete system is $9 \mathrm{~kg}$ and its autonomy is 25 minutes in wind speeds up to $30 \mathrm{~km} / \mathrm{h}$ approximately. A similar system has been presented in [7] for the characterization of cellular and multimode networks. The instrumentation on-board the RPA for the antenna characterization is generally composed by a RX antenna and a spectrum analyzer; the RPA control and telemetry system is composed by a mini-computer, a flight controller and GPS systems. Data are sent to the ground control station, composed by a dedicated computer and several TX/RX systems for the telemetry and measurement data reception.

A system for the characterization of the FF of low frequency antenna arrays has been presented in 8, 9, 10. The instrumentation is slightly different in this example, where the RPA has been equipped with a continuous-wave transmitter (TX) and an antenna which operates as source while the Antenna Under Test (AUT) can register the amplitude data for different positions of the source. Telemetry and flight control instrumentation is similar to the one in the first presented system although in this case, the RPA is equipped with a differential GPS system in order to achieve centimeter positioning accuracy. However, the main limitation of the previous presented systems is that they work in the FF of the AUT and perform direct acquisition of the power emitted by the AUT (i.e. signal strength) for obtaining its radiation pattern and thus, antenna diagnostics cannot be conducted. This limitation has been partially overcome in [11, where the use of navigation and geo-referring systems providing centimeter-level accuracy enable NF acquisition.

Antenna diagnostics [12, 13, 14, 15, 16, is a non-destructive method based on computing an equivalent magnetic currents distribution that, according to the Second Equivalence Principle [17, corresponds to the extremely near field (NF) on the antenna aperture. From the aperture fields antenna diagnostics can be conducted, allowing the detection of failures which prevent the AUT from achieving its expected performance. This is especially interesting for detecting deformations in the antenna structure [14] or antenna array elements malfunction [16]. From the NF acquired data, the FF radiation pattern of the AUT can also be computed by means of NF-FF transformation techniques [18, 19, 20, 21].

In order to perform NF-FF transformations or antenna diagnostics, amplitude and phase information is generally required. Thus, when performing amplitude-only acquisitions, phase retrieval algorithms must be applied.

There are two main approaches for phase retrieval. On the one hand, there are interferometric techniques, 
which are based on creating an interference pattern by means of a known source and the AUT [16, 18]. These techniques are accurate and iteration-free, although they require additional hardware to create the interference pattern. On the other hand, there are iterative techniques, which rely on the information provided by the spatial variation of the NF with distance. They require the acquisition of the field on two or more surfaces [19, 20, 21] and their main limitation is the risk of stagnation due to the use of iterative solvers for non-linear system of equations. In the case of iterative techniques based on the Sources Reconstruction Method [21, the field could be acquired as well in an arbitrary cloud of points around the AUT.

Other important limitation of the existing systems for antenna in-situ characterization based-on RPAs is that it requires using an RPA with high maximum takeoff weight in order to cope with the size and weight of the RF payload [6]. This implies, as well, an increase in the required power and size.

Radio Frequency IDentification (RFID) systems have multiple applications in access control, identification, tracking and monitoring goods, vehicles [22] and persons [23]. These systems consist of RFID tags (active, semi-passive or passive [24],25]), which are inexpensive, and RFID readers, which are significantly more expensive than the former. Among the different RFID standards, in this contribution we will focus on the EPCglobal Gen2 Air Interface Protocol [26] defined for UHF frequency bands, which is also the most widespread and the most suitable for this application. The allocated frequencies for its operation in Europe range from 865.7 to $867.5 \mathrm{MHz}$ in 4 channels. In addition to the extremely low cost of passive RFID tags (euro cents), this technology was selected for this application since the low weight of the tags allows mounting them on a low cost commercial RPA with a limited payload.

Concurrently, RFID technology has been already employed for antenna characterization [27] and gain measurements 28 as well as for indoor tracking and location of RPAs 29] with successful results.

This contribution proposes a novel approach for antenna characterization based on an RFID system. Several tags are fixed to the RPA, avoiding the need for expensive and delicate RF instrumentation onboard, and the RFID reader is connected to the AUT. The reader and the RPA position data are registered while the RPA flights over a set of points surrounding the AUT. The centimeter-level accuracy positioning is achieved using RTK, which is a differential Global Navigation Satellite System (GNSS) that makes use of the GNSS carrier phase [30. In particular, two GNSS have been used: Global Positioning System (GPS) and GLONASS. A post-processing algorithm whose inputs are the RFID reader lectures and the RPA position data, is implemented in order to process the acquired data and perform the phase retrieval and NF-FF transformation to obtain the antenna radiation pattern. The proposed solution allows for in-situ antenna NF phaseless characterization over a non-regular grid with reduced costs at the RFID frequency bands.

The rest of the manuscript is organized as follows: In Section II, the complete system is described in-depth focusing on the instrumentation, the data acquisition process and the developed algorithms for phaseless antenna diagnostics from NF data, and NF-FF transformation. In Section III two application examples are presented for the characterization of a log-periodic antenna array and an RFID antenna array 
in the UHF band. A discussion about the feasibility and performance of the proposed technique is presented in Section IV. Finally, main conclusions are drawn in Section V.

\section{System description}

\subsection{Overview of the measurement system}

The proposed system for antenna characterization based on RFID and RPAs is composed by the following devices:

- An RPA, that includes an array of RFID tags attached to it, and an RTK beacon.

- An RFID reader, which is connected to the AUT.

- A second RTK beacon on a fixed position, which is used as a base station for the RTK system.

- A ground station (e.g. a laptop) connected to the RFID reader. The ground station receives RSS data and RTK positioning information, and processes this information to map RSS measurements with an accuracy of 3-4 cm.

The ground station also runs an iterative phase retrieval technique based on the Sources Reconstruction 90 Method [21] that recovers an equivalent magnetic currents distribution on the aperture plane of the AUT. From this equivalent currents distribution it is possible to identify the radiating sources of the AUT as well as estimating the radiation pattern.

RPA flight path around the AUT can be defined using waypoints. Sometimes waypoints definition is restricted to a minimum distance between two consecutive points. Thus, the RPA can be manually piloted around the AUT in order to acquire sufficient amount of samples of the field radiated by the AUT.

Communication between RPA, RTK beacons, and the ground station can be easily achieved by means of a Wireless Local Area Network (WLAN). For this purpose, a wireless router with a battery is the cheapest and easiest solution. Radio controller works in the $5.8 \mathrm{GHz}$ band.

A block diagram of the system and a flowchart are depicted in Fig. 1]. First, the RFID reader is connected to the AUT and the RPA takes off. The RFID reader sends an interrogation signal that is received by all the RFID tags in the AUT coverage area. In this case, the RFID tags are onboard the RPA. These RFID tags will respond to the RFID interrogation signal, which will be received by the RFID reader. The RFID response signal will be forwarded to the ground station together with a time stamp. In parallel, the RTK base station beacon will forward the corrections that must be applied to the GNSS signal to the RTK beacon placed in the RPA. The latter will use these corrections to improve the position accuracy down to centimeter-level. It will also send the calculated geodetic coordinates (Latitude, Longitude and Height) with 


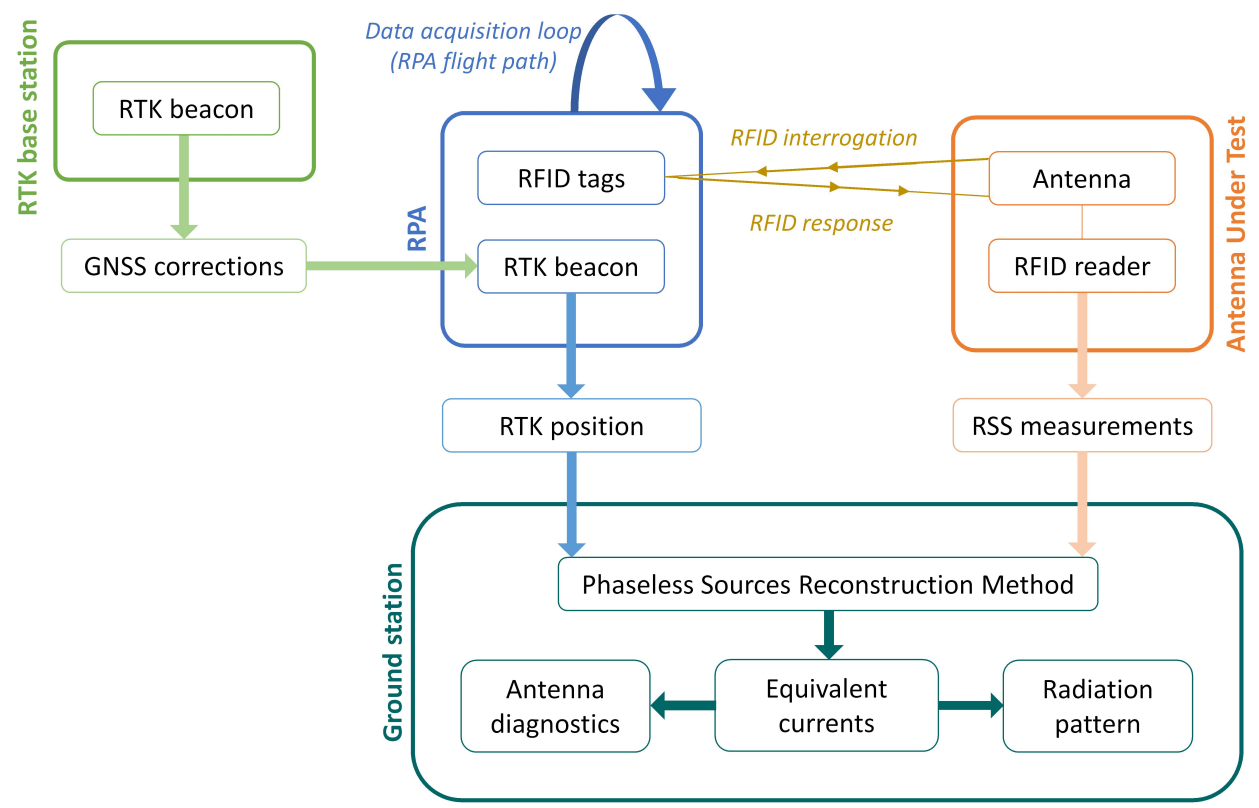

Figure 1: Block diagram of the measurement system.

a time stamp to the ground station. Therefore, RFID measurements can be geo-referred thanks to the fact that both the position information and the RFID measurements have time stamps.

Geo-referred RFID measurements containing signal strength information (RSS) are introduced in the iterative phase retrieval technique based on the Sources Reconstruction Method. From the reconstructed equivalent currents, AUT diagnostics information and AUT radiation pattern are obtained.

A picture of the devices selected for the measurement setup implementation is shown in Fig. 2, The RPA is a Phantom II model that has $400 \mathrm{~g}$ maximum payload weight (close-up picture shown in Fig. 3). Three DogBone RFID tags [31], separated $\lambda / 8$ (4.3 cm at the RFID frequency of $868 \mathrm{MHz})$, are mounted on a plastic and cardboard structure attached to the landing gear of the RPA (see details on Fig. 3). The reason of this layout is to introduce some spatial diversity, taking advantage of the fact that the RPA will be facing the AUT during the flight tests.

Two Reach units from Emlid [32] are used as RTK beacons. One RTK beacon is mounted inside a 3D printed case at the bottom of the RPA and the GNSS antenna is placed in a mast over a ground plane at the top of the RPA, as shown in Fig. 3. The RTK beacon working as base station is placed on a tripod at a known position.

An Impinj Speedway RFID reader is used, connecting it to the ground station (laptop) using an Ethernet cable.

Concerning the RPA flight path, the main restriction is that acquisition points should be spaced less 


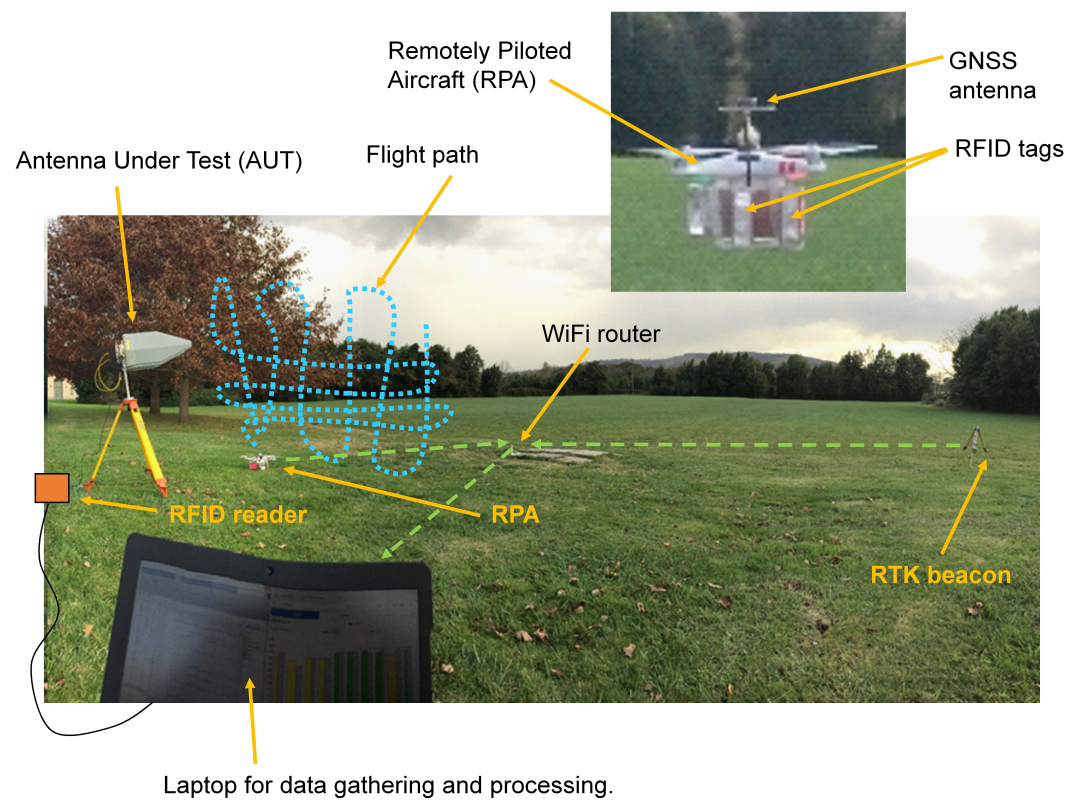

Figure 2: Picture of the implemented measurement system. Dashed lines represent wireless links between the RPA, grounded RTK beacon, and the laptop for data gathering and processing. Dotted line represents RPA flight path.

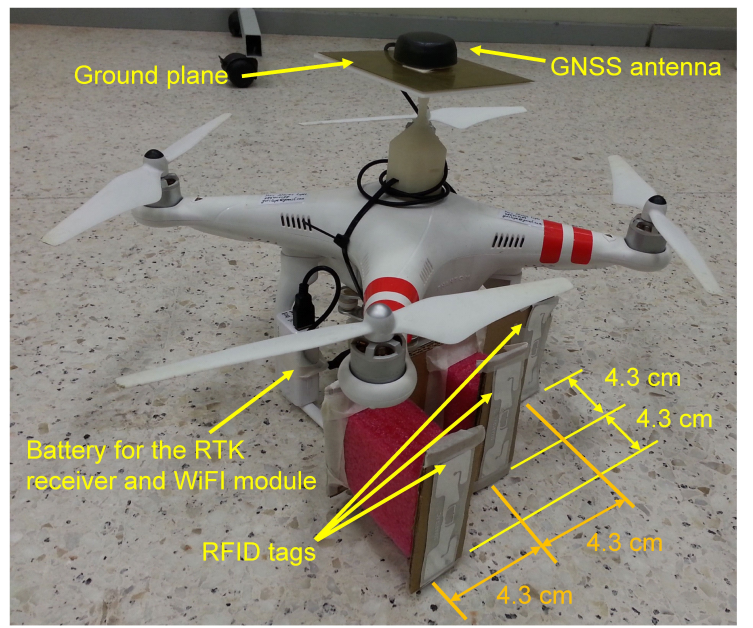

Figure 3: Picture of the RPA (Phantom II model) with the three RFID tags attached and the GNSS antenna on top. 
than $\lambda / 4(8.7 \mathrm{~cm}$ at $868 \mathrm{MHz}$ RFID frequency band). RTK provides centimeter-level positioning accuracy so this condition can be easily fulfilled. The use of waypoints allows defining the flight path in advance. However, the autopilot unit of the RPA chosen for this setup (DJI Phantom 2 [33]) uses an internal GPS unit and the coordinates obtained with the chosen RTK beacons cannot be transferred to this autopilot. Besides, the software tool for waypoints and paths definition 34] does not allow placing waypoints closer than $1 \mathrm{~m}$, which is about $3 \lambda$. Therefore, since RTK information cannot be included for placing waypoints at centimeter-level accuracy, manual flight mode has been selected for the initial tests of the system. Position given by the RTK can be plotted in real-time so the person flying the RPA can visualize the points where RSS samples have been acquired as well as those areas in front of the AUT that have not been measured yet.

The proposed system requires two wireless data links: one for connecting RTK beacons and the ground station, and another for RPA telemetry. As mentioned before, the former works in the $2.4 \mathrm{GHz}$ frequency band, whereas for the selected RPA (DJI Phantom 2) telemetry uses $5.8 \mathrm{GHz}$ frequency band. As RFID operates at $868 \mathrm{MHz}$ (European band), this frequency planning minimizes the risk of electromagnetic interference even when working in the vicinity of the AUT in order to conduct NF measurements.

\subsection{Phaseless antenna diagnostics from near field measurements}

The RFID reader provides, among other parameters, a time stamp of each measurement, the RSS indicator at the RFID reader side, and the phase of the signal backscattered by the detected RFID tags. In this regard, it should be noted that the amplitude and phase values computed by the reader account for both the direct and the return path, so the round-trip propagation path has to be taken into account. In addition, the chip of the Speedway Revolution Reader [35], which is widely employed among other commercial RFID readers, randomly adds 180 degrees to the measured phase values. Hence, it is not possible to know whether the retrieved phase values are the true ones or the true phase values plus 180 degrees.

To avoid this phase ambiguity introduced by the RFID reader, this contribution makes use of an iterative phase retrieval technique based on the SRM for antenna diagnostics and characterization [21]. The idea is to recover an equivalent magnetic currents distribution on the AUT aperture plane, so that they radiate the same field as the AUT.

The flowchart of the iterative phase retrieval technique is depicted in Fig. 4 . It is based on minimizing a cost function $\mathrm{F}$ that relates a vector containing the RSS measurements (i.e. amplitude of the electric field, $|E|)$ taken on the RPA flight path with a vector containing the values of the electric field radiated by the equivalent magnetic currents, $M x, M y$, on the AUT aperture plane, updated at each iteration. An initial guess for the equivalent currents can be set based on a-priori knowledge of the AUT external geometry (e.g. $M x=M y=1$ on the area covering the antenna aperture).

Electric field integral equations relate the equivalent magnetic currents with the radiated electric field 


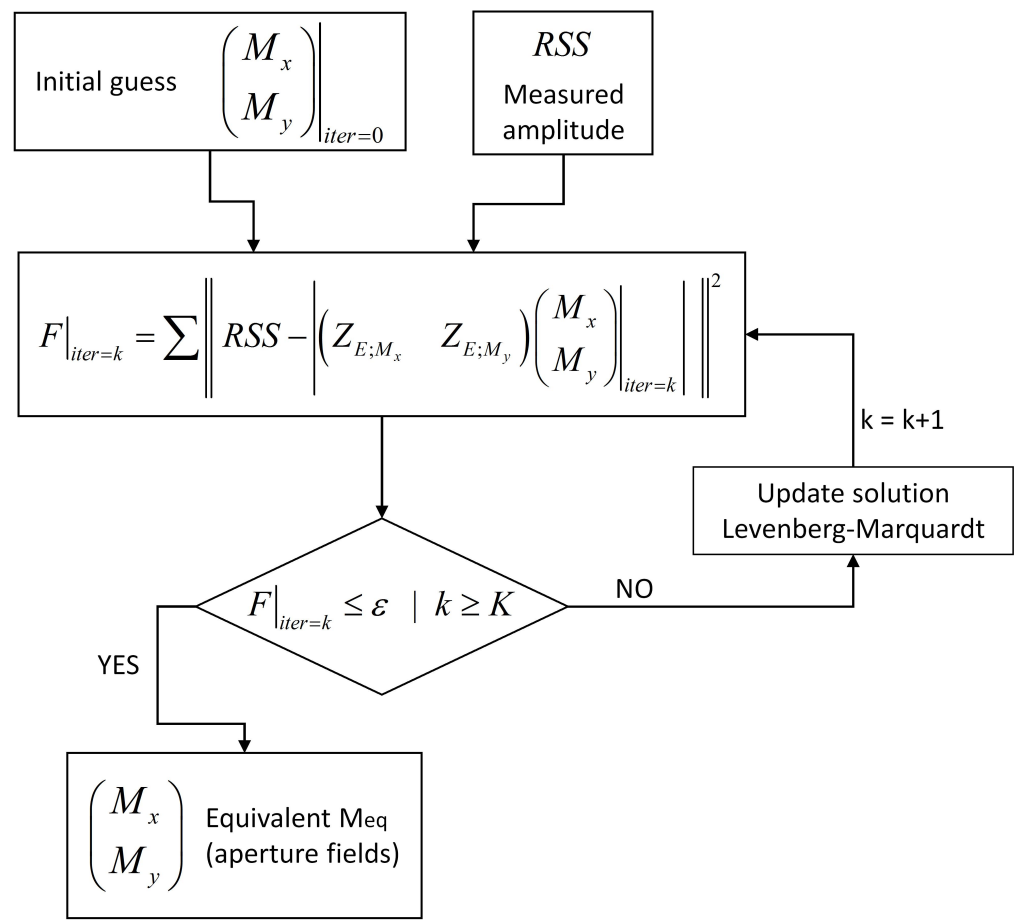

Figure 4: Flowchart of the iterative phase retrieval technique for amplitude-only measurements. 
(Eq. (2) of [21]). As explained in Section II of 21] these integral equations can be numerically evaluated and stored in two matrices, $Z_{E ; M x}$ and $Z_{E ; M y}$, whose size is $\mathrm{N} \mathrm{x} \mathrm{M}$, with $\mathrm{N}$ the number of RSS (electric field) samples, and M, the number of points in which the AUT aperture is discretized. Thus, the multiplication of $\left(Z_{E ; M x} Z_{E ; M y}\right)$ and $(M x ; M y)$ gives the electric field radiated by the equivalent magnetic currents.

Different numerical techniques can be implemented for minimizing the cost function $\mathrm{F}$. In this problem, F is non-linear, so non-linear optimization techniques, such as inexact Newton-Raphson [36] or LevenbergMarquardt [36, must be used. The latter will be considered in this contribution as it has been proved to converge monotonically.

As shown in Fig. 44 at each k-th iteration the cost function $\mathrm{F}$ is evaluated. If the cost function value is equal to or smaller than a certain pre-defined residual $\epsilon$ (typically $\epsilon=0.01$ ) or if the number of iterations $\mathrm{k}$ reaches a pre-defined maximum number of iterations $\mathrm{K}$, the iterative algorithm is stopped. Convergence is achieved when the algorithm stops before reaching the maximum number of iterations $\mathrm{K}$. In that case, it can be stated that the root mean square error of the amplitude of the field radiated by the equivalent currents and the RSS measurements is equal to or less than $\epsilon$.

As mentioned in Section 1 equivalent magnetic currents correspond to the electric field on the antenna aperture [17. The field distribution on the antenna aperture can be used for antenna diagnostics, to identify malfunctioning elements in antenna arrays or deformations in the antenna structure (e.g. reflector antennas [14]). It is worth mentioning again the immediate applicability of a system and method capable of providing in-situ antenna diagnostics.

From the reconstructed equivalent currents, the field at any point of the space in front on the antenna aperture plane can be computed, and hence its radiation pattern [13, 21].

Concerning antenna radiated field sampling requirements, phase retrieval methods require the measured field to be sampled at a rate of, at least, $\lambda / 4$ [16, [18 (in case of amplitude and phase measurements, the sampling rate can be relaxed to $\lambda / 2$ [13). The use of an iterative phase retrieval technique based on the SRM 21 also overcomes the restriction of using a regular acquisition grid. This feature is a key issue in order to perform antenna diagnostics from a set of measurements taken at arbitrary positions, provided these measurements fulfill the aforementioned sampling rates.

\section{Application examples}

\subsection{Example 1: log-periodic antenna array.}

The proposed RFID-based system for in-situ antenna measurement and characterization has been validated by means of the characterization of two different AUTs. The AUT for the first test consists on an array of two log-periodic antennas whose working frequency band ranges from $400 \mathrm{MHz}$ to $3.6 \mathrm{GHz} 37$. 


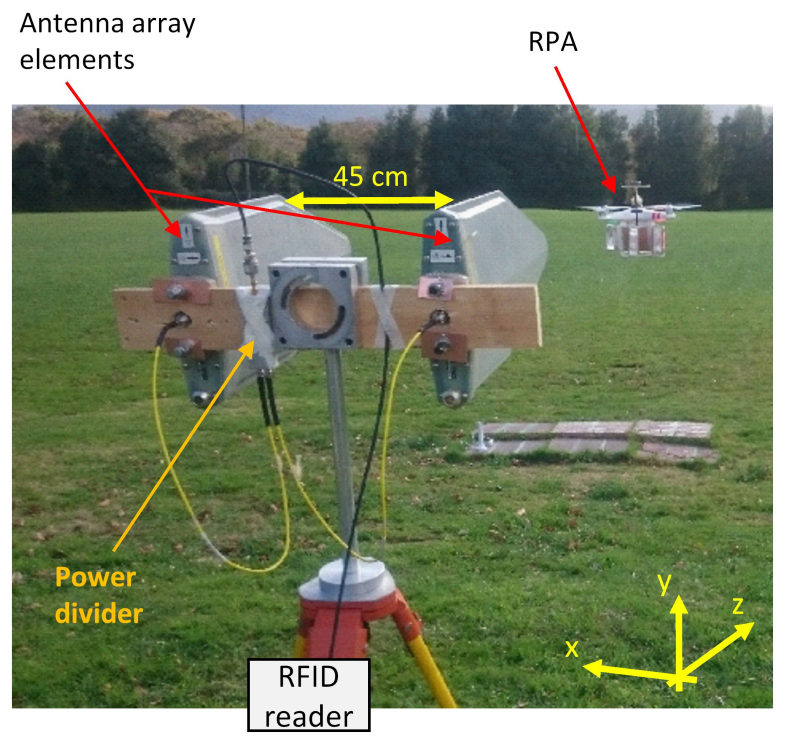

Figure 5: Picture of the log-periodic antenna array.

These antennas are mounted as shown in Fig. 5, so that the separation is $1.3 \lambda$ at $868 \mathrm{MHz}$. These antennas are fed though a power combiner/divider connected to port 1 of the RFID reader.

The flight path is shown in Fig. 6 together with the measured RSS at each position. For each flight path position, three RSS values are collected, each corresponding to the three RFID tags onboard the RPA. Several RSS combination strategies have been tested, such as taking the average or the maximum envelope of the three RSS values, although the way in which RSS values are combined does not impact significantly antenna diagnostics and FF pattern results. An example of measured RSS values is depicted in Fig. 7

It can be noticed that the flight path fits a $5 \mathrm{~m} \times 3 \mathrm{~m}$ aperture placed on average $3 \mathrm{~m}$ in front of the AUT. As the separation between the two log-periodic antennas is $1.3 \lambda$ it can be expected the presence of grating lobes in the radiated field, as observed also in Fig. 6] (grating lobes placed at $\mathrm{x}=-2 \mathrm{~m}$ and $\mathrm{x}=+2$ $\mathrm{m})$. Flight took around 5 minutes, collecting an overall amount of 5500 samples at an average rate of 20 samples per second.

RSS measurements are matched with the positions given by the RTK system using time stamp information. Next, the 5500 geo-referred RSS samples are introduced in the iterative phase retrieval technique based on the SRM. The equivalent magnetic currents are recovered on a $2 \mathrm{~m}$ x $2 \mathrm{~m}$ plane placed on the antenna aperture plane, discretized into $41 \mathrm{x} 41$ points. In this example the Levenberg-Marquardt algorithm stopped after reaching the condition of maximum number of iterations $(\mathrm{K}=30)$ achieving a residual $\epsilon=0.051$ (larger than the targeted residual of $\epsilon=0.01$ ). The error between the measured field amplitude (i.e. RSS values) and the field amplitude radiated by the reconstructed equivalent magnetic currents is depicted in Fig. 8 

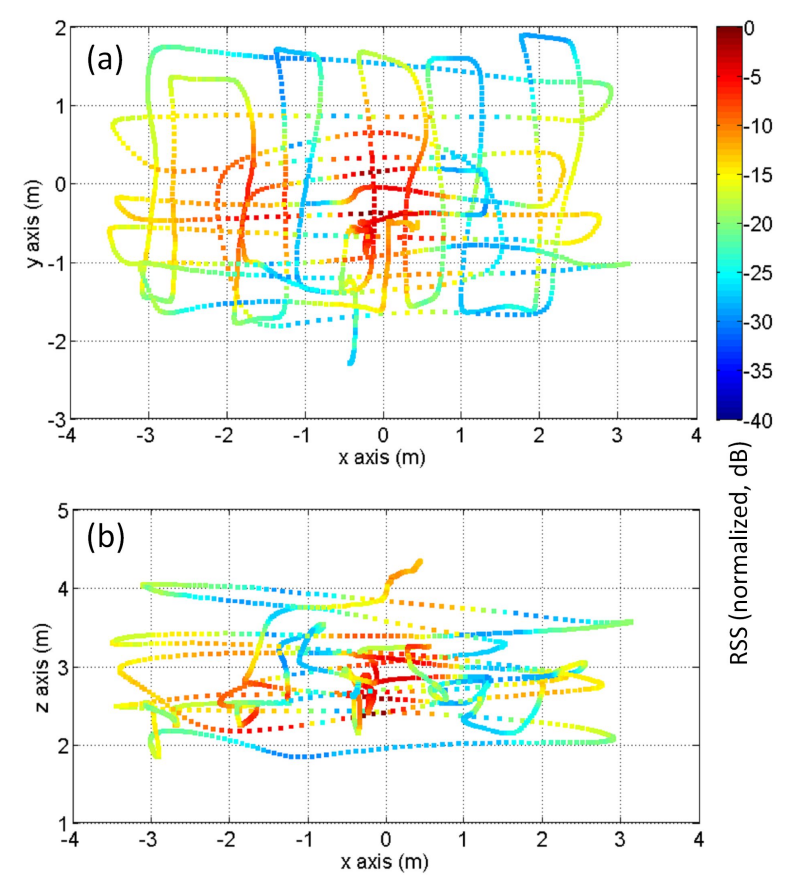

Figure 6: Log-periodic antenna array. Measured RSS levels in the RPA flight path. (a) XY view. (b) XZ view.

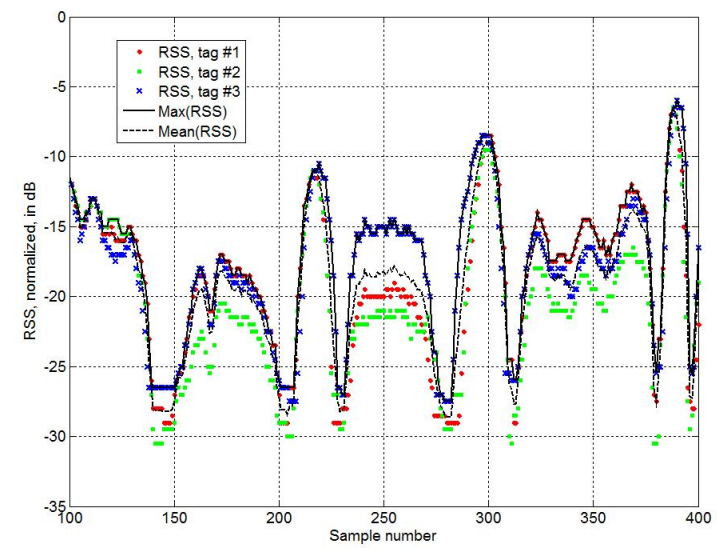

Figure 7: RSS values (300 samples) for the three RFID tags onboard the RPA. Averaged and maximum values are depicted. 


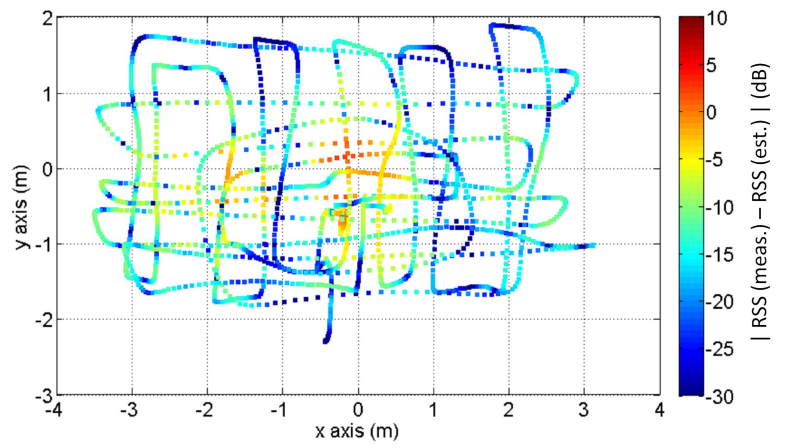

Figure 8: Error (in $\mathrm{dB}$ ) between measured RSS and RSS levels radiated from the reconstructed equivalent currents on the log-periodic antenna array aperture.

For comparison purposes, the log-periodic antenna array has been measured at spherical range in anechoic chamber, using a SH400 probe antenna 38] (Fig. 9). AUT - probe distance is $5.25 \mathrm{~m}$. Measured field has been sampled every 2 degrees in $\theta$ and every 3 degrees in $\phi$.

The amplitude of the equivalent magnetic currents on the aperture plane is plotted in Fig. 10 (a)-(c) considering different ways of combining RSS values from the three RFID tags onboard the RPA. In all the cases, the two log-periodic antennas separated $45 \mathrm{~cm}$ can be identified. Slightly better diagnostics results are obtained (Fig. 10 (c)) when the maximum envelope of the RSS measurements is taken as input in the iterative phase retrieval technique. As a reference, the reconstructed equivalent magnetic currents from anechoic chamber measurements are depicted in Fig. 10 (d). The relative radiation level of each antenna can be also assessed, which is of special interest for detecting malfunctioning elements in antenna arrays. In this case, both antennas are identically manufactured and the feeding is symmetric, so the difference in the aperture fields for each log-periodic antenna is hardly $-2 \mathrm{~dB}$.

From the reconstructed equivalent currents the radiation pattern can be calculated. For this example, the radiation pattern is depicted in Fig. 11 (UV plot) and Fig. 12 ( $\varphi=0^{\circ} \mathrm{c}$ and $\varphi=90^{\circ}$ cuts). Results for RSS measurements (Fig. 11 (a)-(c)) and anechoich chamber measurements (Fig. 11 (d)) are compared: in both cases, the main lobe and the grating lobes can be clearly identified.

Focusing on the FF pattern main cuts (Fig. 12, for $\varphi=0^{\circ}$ the position and width of the main lobe and array factor grating lobes calculated from RSS measurements fit the FF pattern calculated from anechoic chamber measurements. In the case of $\varphi=90^{\circ}$, the radiation pattern of each log-periodic antenna element is not affected by the antenna array factor, so the radiation pattern is less directive. As in the case of antenna diagnostics results, taking the maximum envelope of RSS measurements provides more accurate results than the rest of tested RSS values combinations. 


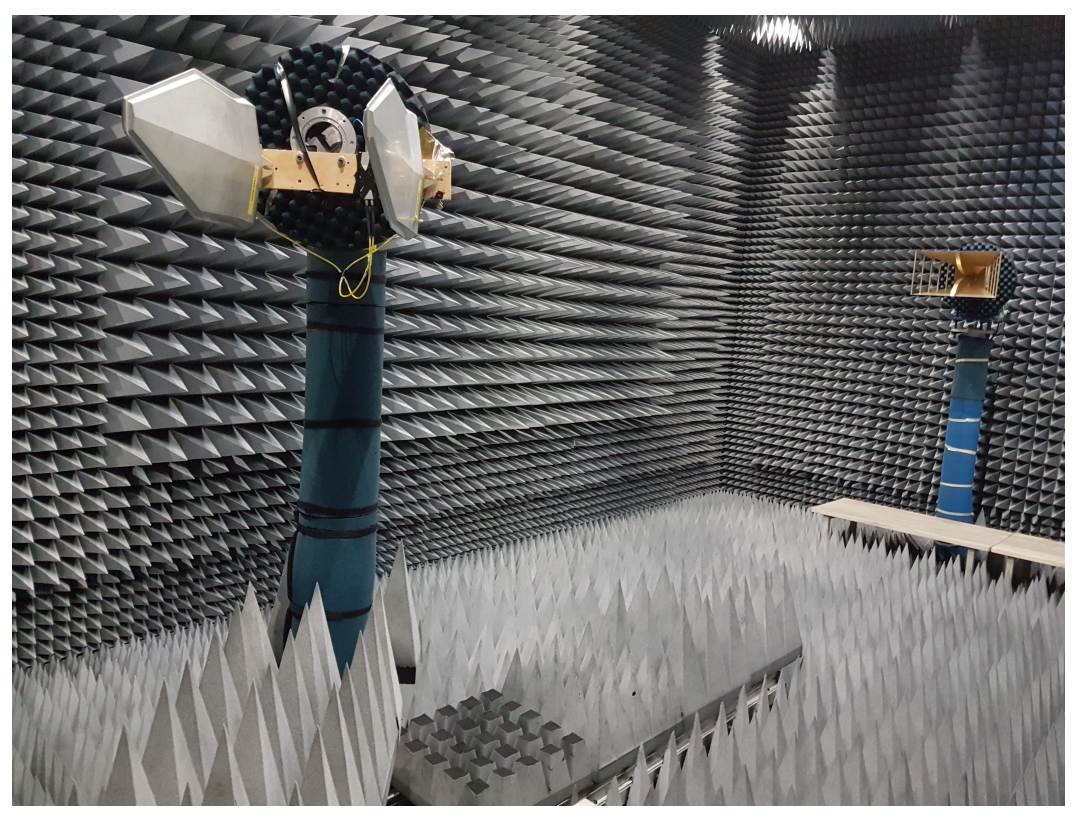

Figure 9: Measurement of the log-periodic antenna array at spherical range in anechoic chamber. Probe antenna SH400 is visible in the right side of the picture.

\subsection{Example 2: RFID antenna array.}

For the second validation example, an array of two UHF RFID antennas [39] has been chosen. These antennas have been placed at different height aiming to test an array of two antennas arbitrary placed. As in Section 3.1 RFID antennas are connected to the port 1 of the RFID reader through a power divider/combiner. For this example 6500 samples have been collected in a 7-minutes flight. RSS measurements and RTK positions are depicted in Fig. 13. A $30 \mathrm{~s}$ video of the RPA flight showing geo-referred RSS measurements in real-time can be watched at https://youtu.be/94hjel_SIDI

Geo-referred RSS measurements have been processed again with the iterative phase retrieval technique based on the SRM, taking the maximum envelope of the three RFID tags onboard the RPA. As in the example of Section 3.1, the equivalent magnetic currents are recovered on a $2 \mathrm{~m}$ x $2 \mathrm{~m}$ planar surface placed on the antenna aperture plane, discretized into $41 \times 41$ points. For this example the Levenberg-Marquardt algorithm stopped again after reaching the maximum number of $\mathrm{K}=30$ iterations with a residual of $\epsilon=0.065$ (larger than the targeted residual of $\epsilon=0.01$ ). The amplitude of the reconstructed equivalent currents as well as a picture of the RFID antenna array is plotted in Fig. 14. It can be noticed that the maximum intensity of the reconstructed equivalent currents fits the position of the RFID antennas. As both antennas are of the same model and the feeding is symmetrical, aperture fields present the same amplitude level.

From the reconstructed equivalent magnetic currents, the antenna array radiation pattern is calculated

(Fig. 15 (a)). It can be noticed that, as the two RFID antennas are spaced more than $1 \lambda$ over the array 

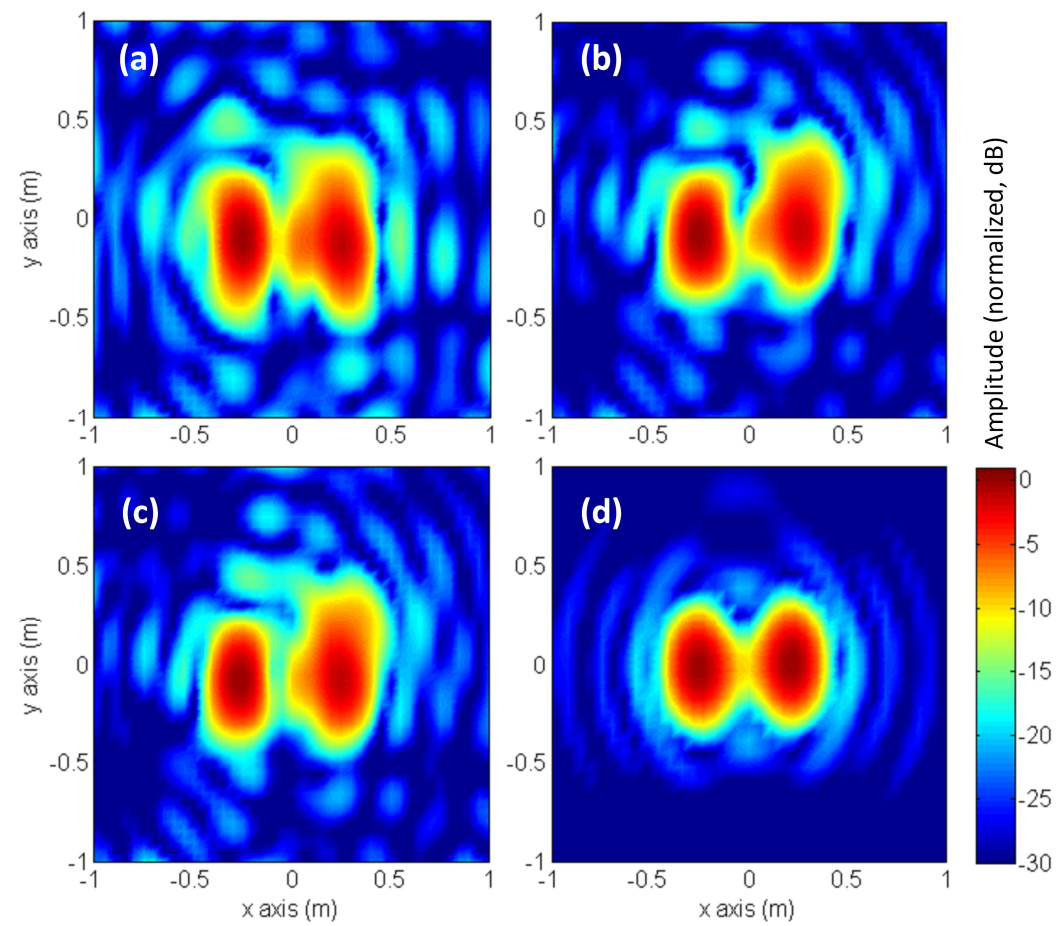

Figure 10: Reconstructed equivalent currents on the log-periodic antenna array aperture. (a) From RSS measurements, one RFID tag. (b) From RSS measurements, maximum evelope of two RFID tags. (c) From RSS measurements, maximum envelope of three RFID tags. (d) From measurements (amplitude and phase information) at spherical range in anechoic chamber. 

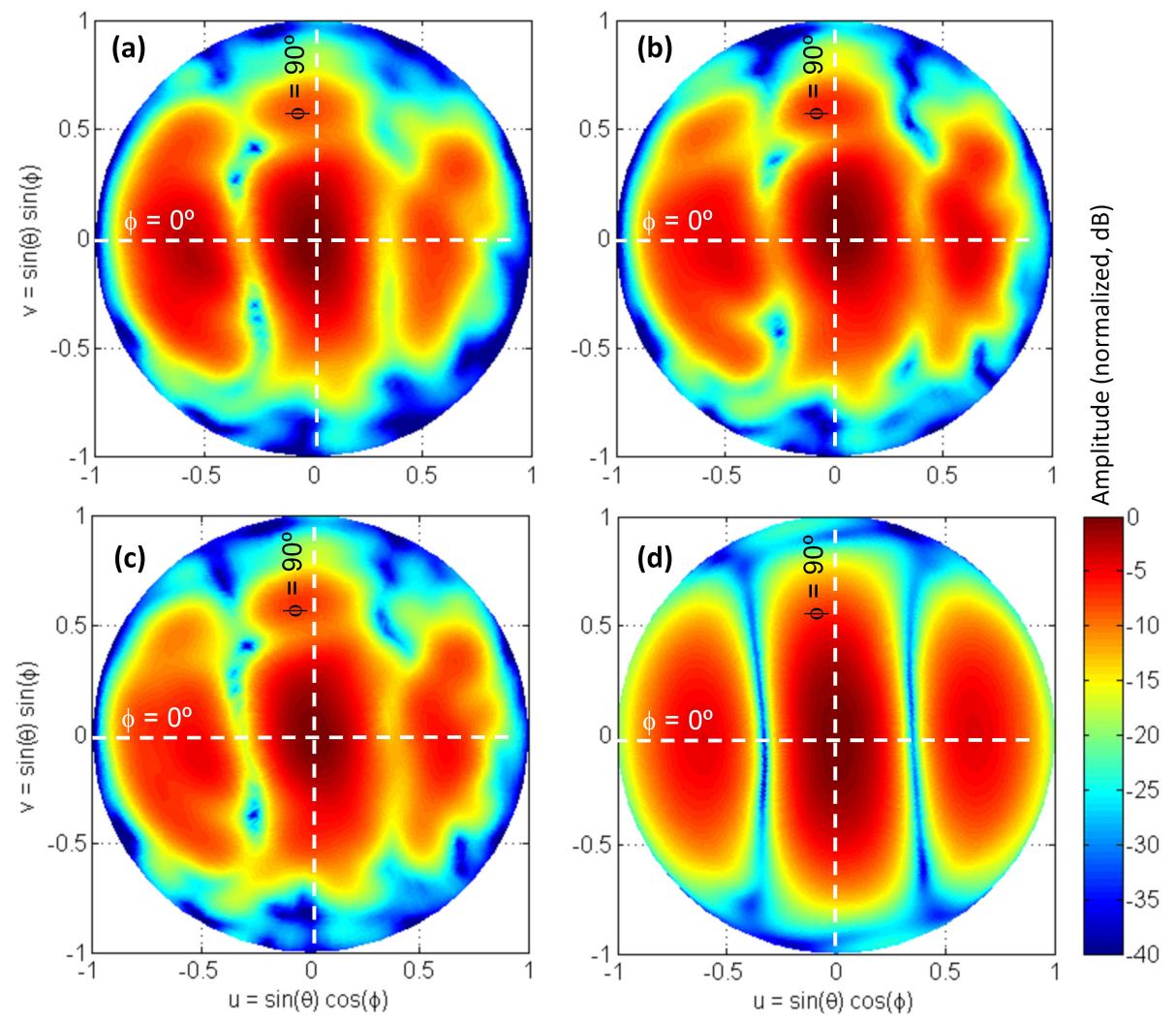

Figure 11: Log-periodic antenna array radiation pattern calculated from the reconstructed equivalent currents using (a) RSS measurements, one RFID tag; (b) RSS measurements, maximum evelope of two RFID tags; (c) RSS measurements, maximum envelope of three RFID tags; and (b) measurements (amplitude and phase information) at spherical range in anechoic chamber. 

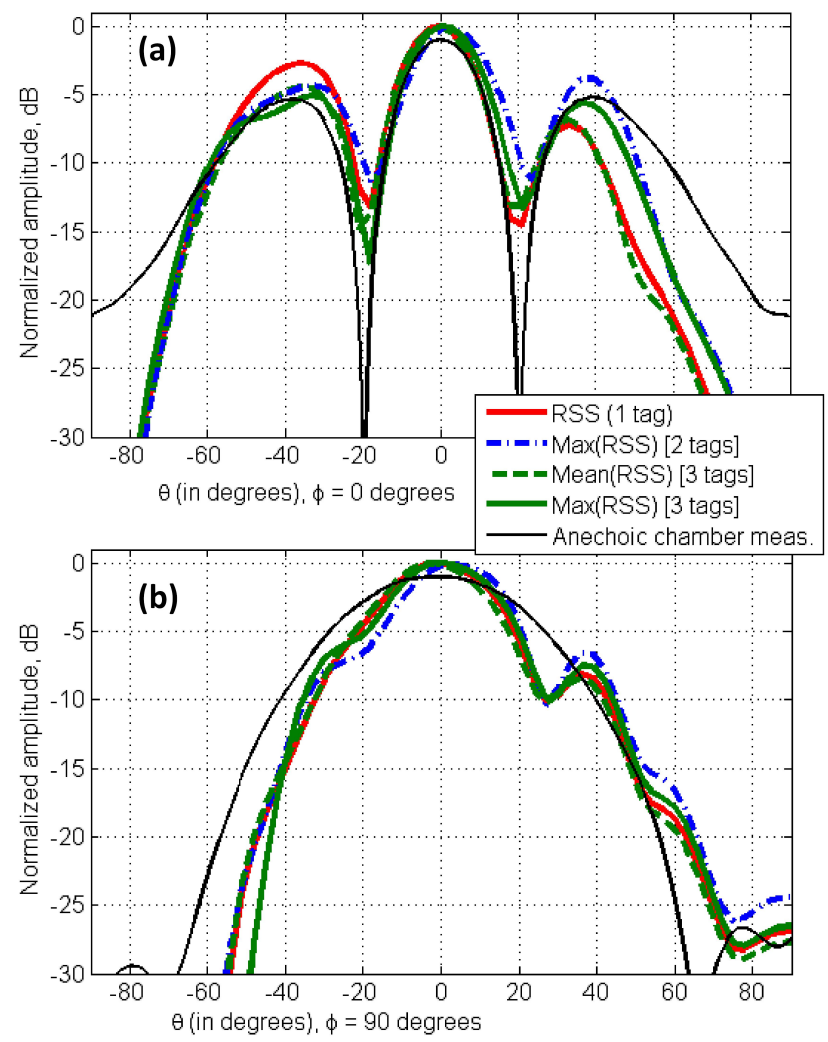

Figure 12: Log-periodic antenna array radiation pattern main cuts. (a) $\varphi=0^{\circ}$. (b) $\varphi=90^{\circ}$. Solid black line: radiation pattern calculated from the reconstructed equivalent currents using RSS measurements. Solid red line: radiation pattern calculated from the reconstructed equivalent currents using RSS measurements (one tag). Dash-dotted blue line: radiation pattern calculated from the reconstructed equivalent currents using RSS measurements (maximum envelope of two tags). Dashed green line: radiation pattern calculated from the reconstructed equivalent currents using RSS measurements (averaging three tags). Solid green line: radiation pattern calculated from the reconstructed equivalent currents using RSS measurements (maximum envelope of three tags). 

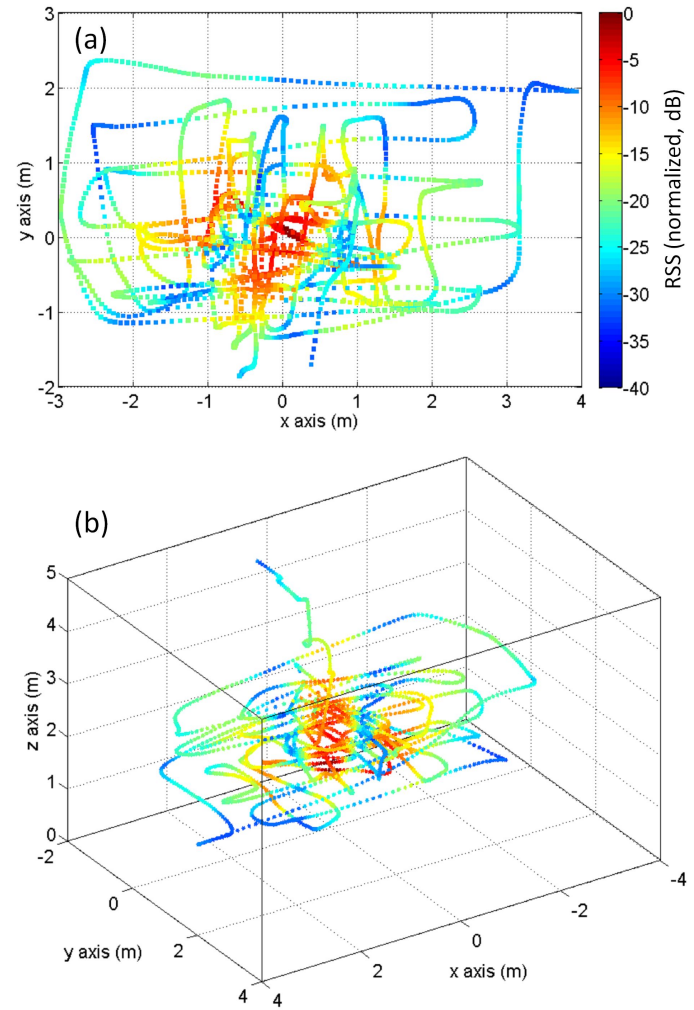

Figure 13: RFID antenna array. Measured RSS levels in the RPA flight path. (a) XY view. (b) 3D view.

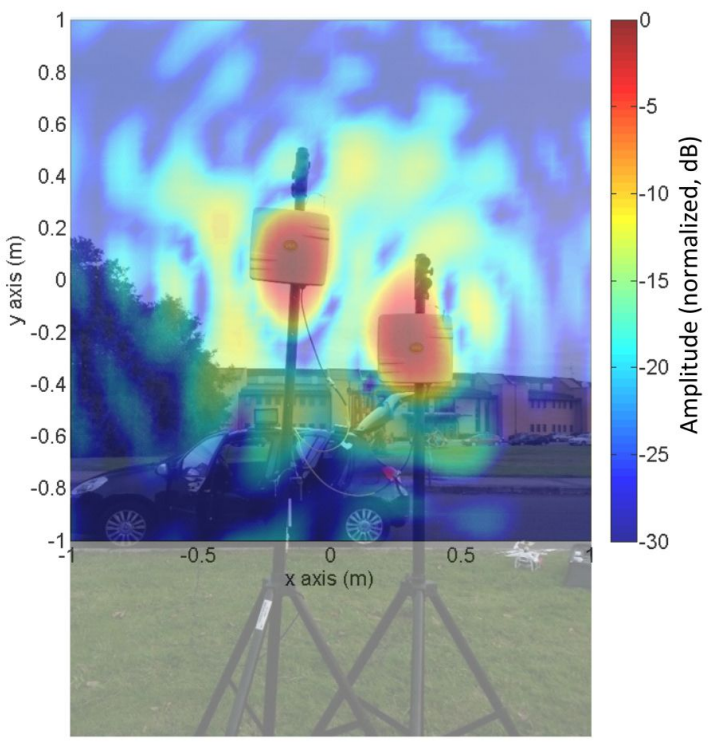

Figure 14: Reconstructed equivalent currents on the RFID antenna array aperture. Picture of the RFID antenna array overimposed. 

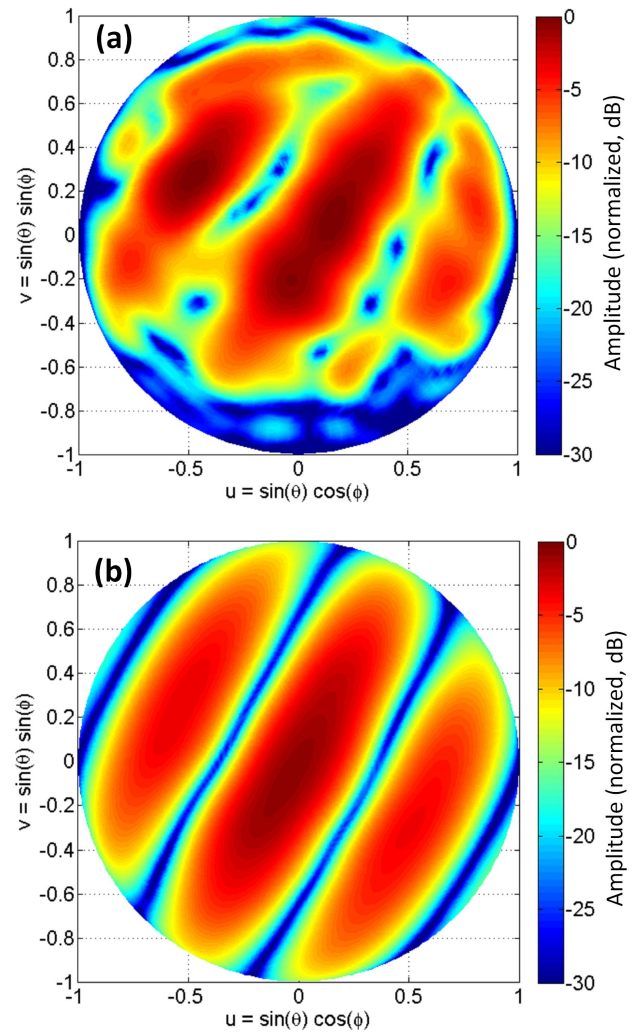

Figure 15: RFID antenna array radiation pattern calculated from the reconstructed equivalent currents using (a) RSS measurements, and (b) measurements (amplitude and phase information) at spherical range in anechoic chamber.

axis, radiation pattern also exhibits grating lobes in such axis (Fig. $15, \phi=-40^{\circ}$ ) similarly to the radiation pattern of the log-periodic antenna array analyzed in Section 3.1. The radiation pattern calculated from measurements at spherical range in anechoic chamber is depicted in Fig. 15 (b), confirming the presence of the aforementioned grating lobes.

\section{Discussion}

The technical specifications and capabilities of the proposed RFID-based system for antenna characterization using RPAs has been compared in Table 1 with those already presented in the literature. In this contribution, the main advantage from a hardware point-of-view is the fact that the RPA payload is reduced thanks to the use of RFID technology, avoiding boarding a receiving probe antenna and a power detector 11. Minimizing the payload weight also enables using smaller RPAs. This issue is of special interest in the case of NF measurements for antenna characterization and diagnostics, as flying larger RPAs in the vicinity of the AUT increases the risk of damaging the AUT in case of an accidental collision. Thus, the use of smaller, low-weight RPAs provides safer operating conditions. Even when flying hundreds of meters 
Table 1: Comparison of antenna measurement techniques using RPAs.

\begin{tabular}{|c|c|c|c|c|}
\hline & $\begin{array}{l}\text { Contribution } \\
\text { [6] }\end{array}$ & $\begin{array}{l}\text { Contributions } \\
{[8,9,10}\end{array}$ & $\begin{array}{l}\text { Contribution } \\
11\end{array}$ & $\begin{array}{l}\text { This contribu- } \\
\text { tion }\end{array}$ \\
\hline Frequency range & $\begin{array}{l}9 \mathrm{kHz}-12 \\
\mathrm{GHz}\end{array}$ & $30-900 \mathrm{MHz}$ & $2-5 \mathrm{GHz}$ & $\begin{array}{l}\text { RFID fre- } \\
\text { quency bands } \\
\text { ( } 868 \mathrm{MHz} \text { in } \\
\text { Europe). }\end{array}$ \\
\hline Positioning system. Accuracy & $\begin{array}{l}\text { GPS. Angular } \\
\text { resolution } 1^{\circ}\end{array}$ & $\begin{array}{l}\text { GPS. Up to } \\
1 \mathrm{~cm} \text { with a } \\
\text { tracking sta- } \\
\text { tion. }\end{array}$ & $\begin{array}{l}\text { RTK and laser } \\
\text { altimeter }(1-2 \\
\mathrm{cm}) \text {. }\end{array}$ & RTK $(3-4 \mathrm{~cm})$. \\
\hline Measurement region & Far field & Far field & $\begin{array}{l}\text { Near field / } \\
\text { Far field }\end{array}$ & $\begin{array}{l}\text { Near field / } \\
\text { Far field }\end{array}$ \\
\hline Radiation pattern uncertainty & $+/-3 \mathrm{~dB}$ & $1 \mathrm{~dB}$ & $+/-4 \mathrm{~dB}$ & $5-6 \mathrm{~dB}$ \\
\hline AUT diagnostics capabilities & None & None & Yes & Yes \\
\hline Onboard receiving unit & Required & Required & Required & Not required \\
\hline
\end{tabular}

away from the antenna as in [6, [8, ,9, [10] for direct FF measurements, piloting larger RPAs requires higher degree of expertise. Furthermore, civil regulations and required licenses for RPA operation in non-controlled airspace are related to RPA take-off weight, typically RPAs $<2 \mathrm{~kg}$, RPAs $<25 \mathrm{~kg}$, and RPAs $>25 \mathrm{~kg}$ [40]. Heavier RPAs require additional licenses and are subject to more restrictive regulations, which eventually impacts on the overall cost of the system.

The main drawback of the proposed system is the fact that the AUT can be tested only at RFID frequency bands, apart from requiring the AUT to be connected to the RFID reader. It must be noticed the higher uncertainty of the proposed technique concerning AUT radiation pattern characterization. This is due to the fact that small measurement uncertainties in the NF (such as multipath propagation, RFID tag pattern) affect the phase retrieval technique and thus the NF-FF transformation, as also happens in 11] when operating in the NF region. In the case of [6], [8, [9], [10, FF pattern is directly measured, so FF uncertainties are mainly due to RPA positioning errors.

\section{Conclusions}

A simple, low-cost in-situ antenna diagnostics system based on RFID technology has been presented. Thanks to the use of RTK, RFID measurements are geo-referred with centimeter-level accuracy, which enables the use of an iterative phase retrieval technique based on the Sources Reconstruction Method to 
recover the electric field distribution on the antenna aperture plane. This distribution provides information about the AUT radiating elements, and it can be also used for AUT radiation pattern calculation.

From the results presented in this contribution, the system has been proved to be quite effective for antenna diagnostics, even when flying the RPA in manual mode without a pre-defined path using waypoints.

Further work will be devoted to integrate RTK information in the RPA flight controller to create waypoints with centimeter-level accuracy, which will provide more accurate acquisition paths, as well as measurements repeatability. Besides, the use of a RFID reader capable of providing phase without $180^{\circ}$-ambiguity will be also assessed enabling direct acquisition of the phase of the radiated field.

\section{Acknowledgements}

This work has been supported by the Ministerio de Economa y Competitividad - Gobierno de España under projects TEC 2014-54005-P (MIRIIEM), MINECO-15-TEC2014-55290-JIN (PORTEMVISION); under grants FPU15/06341 and FPU15/06431; and by the Gobierno del Principado de Asturias through the PCTI 2013-2017, FC-15-GRUPIN14-114. This work has been developed under the framework of the Universidad de Oviedo postdoctoral degree Expert in Remotely Piloted and Autonomous Flight Aircrafts (wWw.dronesuniovi.es).

\section{References}

[1] D. Hausamann, W. Zirnig, G. Schreier, P. Strobl, Monitoring of gas pipelines a civil uav application, Aircraft Engineering and Aerospace Technology 77 (2005) 352-360.

[2] Y. Ham, K. K. Han, J. J. Lin, M. Golparvar-Fard, Visual monitoring of civil infrastructure systems via camera-equipped unmanned aerial vehicles (UAVs): a review of related works, Visualization in Engineering 4 (2016) 1-8.

[3] G. J. Grenzdorffer, A. Engel, B. Teichert, The photogrammetric potential of low-cost UAVs in forestry and agriculture, Vol. XXXVII Part B1, The international archives of the photogrammetry, remote sensing and spatial information sciences, Beijing, 2008.

[4] E. Honkavaara, H. Saari, J. Kaivosoja, I. Polonen, T. Hakala, P. Litkey, J. Makynen, L. Pesonen, Processing and assessment of spectrometric, stereoscopic imagery collected using a lightweight uav spectral camera for precision agriculture, Remote Sensing 5 (2013) 5006-5039.

[5] R. Salvini, G. Mastrorocco, G. Esposito, S. D. Bartolo, J. Coggan, C. Vanneschi, Use of a remotely piloted aircraft system for hazard assessment in a rocky mining area (lucca, italy), Natural Hazards and Earth System Sciences $\mathrm{n} / \mathrm{a}$ (in review (2017)) 1-35.

[6] J. Schreiber, Antenna pattern reconstitution using unmanned aerial vehicles (UAVs), in: IEEE Conference on Antenna Measurements and Applications (CAMA), 2016, pp. 1-3.

[7] E. Teng, J. D. Falcao, C. R. Dominguez, F. Mokaya, P. Zhang, B. Iannucci, Aerial sensing and characterization of three-dimensional RF fields, in: Second International Workshop on Robotic Sensor Networks, 2015.

[8] G. Virone, F. Paonessa, A. Tibaldi, Z. Farooqui, G. Addamo, O. A. Peverini, G. Naldi, UAV-based radiation pattern verification for a small low-frequency array, in: IEEE Antennas and Propagation Society International Symposium (APSURSI), 2014, pp. 995-996. 
[9] F. Paonessa, G. Virone, I. Aicardi, A. M. Lingua, M. Piras, P. Maschio, P. Bolli, G. Addamo, O. A. Peverini, R. Orta, R. Tascone, Recent results in antenna pattern measurement with UAVs, in: 2015 International Conference on Electromagnetics in Advanced Applications (ICEAA), 2015, pp. 720-721.

[10] G. Virone, A. M. Lingua, M. Piras, A. Cina, F. Perini, J. Monari, F. Paonessa, O. A. Peverini, G. Addamo, R. Tascone, Antenna pattern verification system based on a micro unmanned aerial vehicle (UAV), IEEE Antennas and Wireless Propagation Letters 13 (2014) 169-172.

[11] M. Garcia-Fernandez, Y. Alvarez, A. Arboleya, B. Gonzalez-Valdez, Y. Rodriguez-Vaqueiro, M. E. de Cos, F. Las-Heras, Antenna diagnostics and characterization using unmanned aerial vehicles, IEEE Access (2017) 1-12.

[12] Y. Rahmat-Samii, Surface diagnosis of large reflector antennas using microwave holographic metrology: an iterative approach, Radio Science 19 (1984) 1205-1217.

[13] Y. Alvarez, F. Las-Heras, M. Pino, Reconstruction of equivalent currents distribution over arbitrary three-dimensional surfaces based on integral equation algorithms, IEEE Transactions on Antennas and Propagation 55 (12) (2007) 3460-3468.

[14] Y. Alvarez, C. Cappellin, F. Las-Heras, O. Breinbjerg, On the comparison of the spherical wave expansion-to-plane wave expansion and the sources reconstruction method for antenna diagnostics, Progress In Electromagnetics Research 87 (2008) $245-262$.

[15] F. J. Cano-Facila, S. Burgos, F. Martin, M. Sierra-Castaner, New reflection suppression method in antenna measurement systems based on diagnostic techniques, IEEE Transactions on Antennas and Propagation 49 (2011) 941-949.

[16] J. Laviada, A. Arboleya, Y. Alvarez, C. Garcia, F. Las-Heras, Phaseless antenna diagnostics based on off-axis holography with synthetic reference wave, IEEE Antennas and Wireless Propagation Letters 13 (2014) 43-46.

[17] C. Balanis, Advanced Engineering Electromagnetics, Wiley, New York, 1989.

[18] A. Arboleya, J. Laviada, J. Ala-Laurinaho, Y. Alvarez, F. Las-Heras, A. Raisanen, Phaseless characterization of broadband antennas, IEEE Transactions on Antennas and Propagation 64 (2) (2016) 484-495.

[19] S. Razavi, Y. Rahmat-Samii, Phaseless measurements over nonrectangular planar near-field systems without probe corotation, IEEE Transactions on Antennas and Propagation 61 (1) (2013) 143-152.

[20] S. Razavi, Y. Rahmat-Samii, A new look at phaseless planar near-field measurements: Limitations, simulations, measurements, and a hybrid solution, IEEE Antennas and Propagation Magazine 49 (2) (2007) 170-178.

[21] Y. Alvarez, F. Las-Heras, M. Pino, The sources reconstruction method for amplitude-only field measurements, IEEE Transactions on Antennas and Propagation 58 (8) (2010) 2776-2781.

[22] F. Lazzari, A. Buffi, P. Nepa, S. Lazzari, Numerical investigation of an uwb localization technique for unmanned aerial vehicles in outdoor scenarios, IEEE Sensors Journal 17 (2017) 2896-2903.

[23] Y. Alvarez, M. E. de Cos, F. Las-Heras, A received signal strength RFID-based indoor location system, Sensors and Actuators A: Physical 255 (2017) 118-133.

[24] F. Chetouane, An overview on RFID technology instruction and application, IFAC-PapersOnLine 48 (3) (2015) 382-387.

[25] J. F. Salmeron, A. Rivadeneyra, M. Agudo-Acemel, L. F. Capitn-Vallvey, J. Banqueri, M. A. Carvajal, A. J. Palma, Printed single-chip uhf passive radio frequency identification tags with sensing capability, Sensors and Actuators A: Physical 220 (2014) 281-289

[26] GS1, EPC radio-frequency identity protocols generation-2 UHF RFID (11 2016). URL http://www.gs1.org/sites/default/files/docs/epc/Gen2_Protocol_Standard.pdf

[27] L. W. Mayer, A. L. Scholtz, Simple and accurate radiation pattern measurement of UHF RFID transponders, in: International Symposium on Antennas and Propagation, 2008.

[28] P. Nikitin, K. V. S. Rao, Gain measurement of antennas using RFID, in: IEEE International Symposium on Antennas and Propagation (APS URSI), 2011, pp. 1-4.

[29] J. S. Choi, B. R. Son, H. K. Kang, D. H. Lee, Indoor localization of unmanned aerial vehicle based on passive UHF 
RFID systems, in: 9th IEEE International Conference on Ubiquitous Robots and Ambient Intelligence (URAI), 2012, pp. $188-189$.

[30] A. El-Rabbany, Introduction to GPS: the Global Positioning System, Artech House, London, 2002.

[31] Smartrac DogBone RFID inlays and tags (11 2016).

URL https://www . smartrac-group. com/dogbone.html

[32] Reach RTK from Emlid (11 2016). URL https://emlid.com/reach/

[33] DJI phantom 2 drone (11 2016).

URL https://www.dji.com/phantom-2

[34] DJI litchi for phantom 2 (3 2017).

URL https://play .google.com/store/apps/details?id=com . aryuthere $\cdot$ visionplus2\&hl=es

[35] Impinj speedway R420 RFID reader (11 2016). URL https ://support.impinj . com/hc/en-us/articles/202755298-Reader-Documentation

[36] Numerical recipes in c: the art of scientific computing, Cambridge University Press, 1988-1992, pp. 379-383 (NewtonRaphson), 681-688 (Levenberg-Marquardt).

[37] Rohde \& schwarz hl040 log-periodic antenna (2 2017).

URL https://cdn.rohde-schwarz.com/pws/dl_downloads/dl_common_library/dl_brochures_and_datasheets/pdf_1/ HL040_catalog_2013_90_91.pdf

[38] Probe antenna SH400 from MVG (10 2017). URL http://www.satimo.com/sites/www.satimo.com/files/Dual_ridge_horns_BD3.pdf

[39] CAEN RFID WANTENNAX019 (11 2016).

URL http://www . caenrfid.it/en/CaenProd.jsp?parent=107\&idmod=835\#

[40] Spanish aviation safety and security agency AESA, RPA regulation (2 2017).

URL http://www.seguridadaerea.gob.es/LANG_EN/cias_empresas/trabajos/rpas/default.aspx 